\title{
Iodinated contrast media allergy is rare in patients undergoing sialography
}

\author{
Chen Nadler, ${ }^{1}$ Andra Rettman, ${ }^{1}$ Nufar Benvenisty, ${ }^{1}$ Yaarit Ribak, ${ }^{2}$ Alon Y. Hershko, ${ }^{2 *}$ Yuval Tal ${ }^{2 *}$
}

\begin{abstract}
Background: Iodinated contrast media allergy is considered as a strong contra-indication for performing sialography. There is little evidence to support this approach.

Objective: To evaluate the rate of iodinated contrast media (ICM) allergy in subjects undergoing sialography and to assess the risk for allergic responses in patients with a previous diagnosis of allergy.

Methods: We retrospectively reviewed sialo-CBCT studies performed from 2014 to 2019. During the study period we implemented a protocol for performing sialo-CBCT in patients with a prior diagnosis of allergy: 1) Clinical data were collected from a questionnaire and medical records. 2) No premedication was administered but, instead, oxygen, epinephrine and a resuscitation cart were accessible. 3) Following the procedure, each patient was observed for one hour and contacted by telephone 24 hrs later.
\end{abstract}

Results: No allergic responses were documented in the medical records of 1515 subjects following sialo-CBCT studies, including 13 individuals previously diagnosed with ICM allergy. Investigation of the subgroup with prior allergy disclosed that the range of injected volume was between $2 \mathrm{ml}$ to $6.2 \mathrm{ml}$ per patient and that complete secretion of ICM was detected in 7 of 13 patients. In the remainder of subjects, retention rates of 5-50\% were observed.

Conclusions: Allergic reactions are exceedingly rare following sialo-CBCT studies regardless of a previous diagnosis of allergy. Pre-medication with corticosteroids and antihistamines is usually not warranted.

Key words: iodinated contrast media; drug hypersensitivity; sialography; iodine allergy; salivary glands

\section{From:}

${ }^{1}$ Maxillofacial Imaging, Department of Oral Medicine,

School of Dental Medicine

2 Allergy and Clinical Immunology Unit, Department of Medicine,

Hadassah-Hebrew University Medical Center, Jerusalem, Israel

* These authors equally contributed to this work

\section{Introduction}

Sialography is a simple procedure that allows imaging of both the structure and the function of the major salivary glands. ${ }^{1}$ It is instrumental in the diagnosis and treatment of non-tumoral salivary pathologies which may be obstructive, autoimmune or a combination of both. ${ }^{2,3}$ Imaging is obtained by injection of $2-5 \mathrm{ml}$ of water-soluble iodinated contrast media (ICM) through the orifice of the Stenson's or Warthon's salivary duct, belonging to the parotid and submandibular glands, respectively. A cone-beam computerized tomography (CBCT) study is then performed to create a volumetric image with increased spatial resolution and a relatively low dose of radiation, thus constituting a sialo-CBCT examination., ${ }^{4,5}$

\author{
Corresponding author: \\ Alon Y. Hershko \\ President, Israeli Association of Allergy and Clinical Immunology \\ Head, Department of Medicine C \\ Hadassah-Hebrew University Medical Center, \\ Ein Kerem, Jerusalem, Israel 91120 \\ E-mail: alonh@hadassah.org.il
}

The catheter is subsequently removed and 5 minutes later an additional image is obtained to examine glandular function. ICM is then secreted to the oral cavity and is swallowed by the patient. ${ }^{4}$ This usually results in only negligible systemic absorption. In some subjects ICM may be retained within the gland or extravasate to the glandular parenchyma, due to an iatrogenic factor or impaired cell-to-cell adhesion.

ICM have been recognized decades ago as allergenic agents. Cases of severe anaphylactic responses have been well documented over the years, with potential life-threatening outcomes. The mechanisms underlying these responses are not entirely clear and include rare IgE-dependent mast cell 
degranulation as well as the more prevalent non-IgE mediated reactions. The latter may be explained by direct mast cell stimulation due to the hyperosmolar properties of the injected media. Of note, the advent of new-generation ICM has considerably decreased the rate of allergic responses thus prompting the reassessment of this adverse effect. Although risk factors have been reported, they increase the likelihood of a reaction by less than one order of magnitude. Patients with a prior reaction disclose a 5 -fold increase of developing a future allergic event. Other risk factors have been reported as well, although none of them require restriction of ICM use or premedication due to their modest impact. Examples for such conditions include unrelated allergies, asthma, cardiac disease, anxiety, middle age, beta blocker drugs as well as several others. ${ }^{6}$

The possibility of systemic exposure to ICM following sialography has raised the concern of allergic reactions. Sialography is traditionally regarded as contraindicated, and almost invariably avoided, in patients with a previous diagnosis of ICM allergy. ${ }^{7-9}$ Strikingly, there are little, if any, published data to support an increased risk for sialography-related reactions in individuals with previous ICM allergy. Furthermore, it is not clear whether a previous diagnosis of ICM allergy predicts adverse outcomes during this procedure.

\section{Methods}

In this communication we retrospectively report our experience with sialo-CBCT studies performed from 2014 to 2019 including an account of the outcome in subjects with previously reported ICM allergy. Data was retrieved by the screening of an archive that contains all the sialo-CBCT records within the Department of Oral Medicine. All subjects were included without any exclusion. In this study, allergy was defined as a systemic reaction that occurs within one hour from exposure to ICM, and involves one or more of the typically involved organ-systems (skin, gastrointestinal tract as well as respiratory, nervous and circulatory systems). Transient flushing was not considered as allergy.

We devised a protocol for performing sialo-CBCT in allergic patients, in collaboration between the Department of Oral Medicine, Sedation and Maxillofacial Imaging and the Unit of Allergy and Clinical Immunology at the Hadassah University Medical Center (Jerusalem, Israel) (Figure 1). First, clinical data were obtained through a questionnaire and review of medical records. Next, sialography was carried out without premedication but, instead, an intravenous line was placed prior to ICM injection, as a precaution. Additionally, oxygen, epinephrine and a resuscitation cart were accessible. Following the procedure, each patient was observed for one hour and contacted $24 \mathrm{hrs}$ later by telephone.

CBCT imaging is performed using the I-CAT CBCT system (Imaging Sciences International, Hatfield, PA) or with 3D Accuitomo 170 (J. Morita Corp., Osaka, Japan). Sialography was done with the non-ionic, water soluble, low-osmolality agent Iomeron 350 (Bracco, UK).

Access to medical records for the purpose of this study was approved by the local Institutional Ethics Committee of Hadassah Medical Center in keeping with the principles of the Declaration of Helsinki (application number HMO-0292-19).

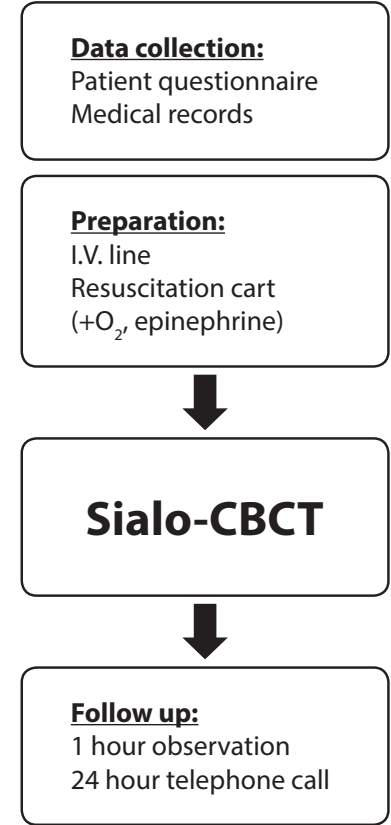

Figure 1. Protocol for sialo-CBCT in patients with a history of ICM allergy.

Prior to the procedure, demographic and clinical data were collected as well as details on previous allergic responses to ICM. Patients were not instructed to take pre-medication. Instead, preparations were done for treatment of potential anaphylactic reactions, including placement of an intravenous line and preparation of resuscitation equipment. Following the procedure, patients were observed for one hour and then contacted by telephone after 24 hours.

\section{Result}

Screening of medical records yielded a total of 1,515 reports of Sialo-CBCT studies conducted in the course of the study period, disclosing no allergic reactions during or after the procedure. This observation includes both immediate and late hypersensitivity reactions.

The study population of included 13 individuals previously diagnosed with ICM allergy, with ages ranging from 25 to $76 \mathrm{yrs}$, and a female preponderance (F:M ratio 11:2; Table 1). Review of patient medical records disclosed that the most common finding that had led to the diagnosis of ICM allergy was rash $(\mathrm{N}=4)$ followed by palpitations $(\mathrm{N}=1)$ and gastrointestinal symptoms $(\mathrm{N}=1)$. In 5 patients, no details could be identified on findings that could justify the diagnosis of ICM allergy. In two additional subjects the documented findings were not suggestive of allergy. One of them had not experienced a reaction but had a family history of anaphylaxis, while the other reported fever only. Of note, none of the medical records disclosed a previous severe of life-threatening allergic response. The indications for sialography were investigation of mouth dryness $(\mathrm{N}=10)$, salivary gland swelling $(\mathrm{N}=2)$ and pain $(\mathrm{N}=1)$. The parotid gland was examined in the majority of cases $(\mathrm{N}=11)$ followed by the submandibular glands $(\mathrm{N}=2)$. An intravenous line was placed in all patients in preparation for the procedure. In spite of our recommendations, three patients were given premedication by their primary care physicians prior to sialography. 
Table 1. Characteristics of subjects with previously reported ICM allergy who underwent sialography

\begin{tabular}{|c|c|c|c|c|c|c|c|c|}
\hline & $\begin{array}{l}\text { Gender/ } \\
\text { Age }\end{array}$ & $\begin{array}{l}\text { Previous reaction to } \\
\text { ICM }\end{array}$ & $\begin{array}{l}\text { Indication for } \\
\text { sialo-CBCT }\end{array}$ & Gland & $\begin{array}{l}\text { preparation } \\
\text { for sialography }\end{array}$ & $\begin{array}{l}\text { ICM volume, } \\
\text { ml right; left }\end{array}$ & $\begin{array}{c}\text { ICM } \\
\text { secretion (\%) }\end{array}$ & $\begin{array}{l}\text { sialo-CBCT } \\
\text { diagnosis }\end{array}$ \\
\hline 1 & $\mathrm{~F} / 25$ & $\begin{array}{l}\text { family history of } \\
\text { anaphylaxis }\end{array}$ & pain & parotid & IV line ${ }^{*}$ & $1.5 ; 1.8$ & 100 & $\begin{array}{c}\text { juvenile recurrent } \\
\text { parotitis }\end{array}$ \\
\hline 2 & $\mathrm{~F} / 44$ & $\begin{array}{c}\text { palpitations, } \\
\text { diaphoresis, tremor }\end{array}$ & dryness & parotid & IV line & $1.2 ; 1$ & 100 & normal findings \\
\hline 3 & $\mathrm{M} / 47$ & rash & dryness & parotid & IV line & $1 ; 0$ & 100 & atrophic gland \\
\hline 4 & $\mathrm{~F} / 49$ & nausea, diarrhea & dryness & parotid & IV line & $2 ; 2$ & 100 & sialadenitis \\
\hline 5 & $\mathrm{~F} / 49$ & N/A & swelling & sub-mandibular & IV line & N/A; 0 & 80 & sialadenitis \\
\hline 6 & $\mathrm{~F} / 50$ & N/A & dryness & parotid & IV line & $2 ; 2$ & 80 & sialadenitis \\
\hline 7 & $\mathrm{~F} / 55$ & N/A & dryness & parotid & IV line ${ }^{*}$ & $1.5 ; 1.5$ & 95 & normal findings \\
\hline 8 & $\mathrm{~F} / 56$ & rash & swelling & sub-mandibular & IV line $e^{\star *}$ & $0 ; 2$ & 100 & normal findings \\
\hline 9 & $\mathrm{~F} / 62$ & fever & dryness & parotid & IV line & $1.5 ; 1.8$ & $80(\mathrm{R}), 50(\mathrm{~L})$ & sialadenitis \\
\hline 10 & $\mathrm{~F} / 63$ & rash & dryness & parotid & IV line & $1 ; 1$ & 100 & sialadenitis \\
\hline 11 & $\mathrm{M} / 65$ & N/A & dryness & parotid & IV line & $3.2 ; 3$ & 100 & normal findings \\
\hline 12 & $\mathrm{~F} / 74$ & rash & dryness & parotid & IV line & $1 ; 1$ & 100 & normal findings \\
\hline 13 & $\mathrm{~F} / 76$ & N/A & dryness & parotid & IV line & $1 ; 2.5$ & $\begin{array}{c}95 \\
\text { extra-vasation }\end{array}$ & sialadenitis \\
\hline
\end{tabular}

* prednisone and-anti histamine; ${ }^{* *}$ antihistamine; N/A: not available;

In these subjects, premedication included a combination of anti-histamines and corticosteroids $(\mathrm{N}=2)$ or anti-histamine only $(\mathrm{N}=1)$. The range of injected volume was between $2 \mathrm{ml}$ to $6.2 \mathrm{ml}$ per patient, and complete secretion of ICM was detected in 7 of 13 patients. In the remainder of subjects, retention rates of $5-50 \%$ were observed, while extravasation occurred in one examination only (Figure 2). The findings that were identified by sialography were sialadenitis $(\mathrm{N}=6)$, normal glands $(\mathrm{N}=5)$, juvenile recurrent parotitis $(\mathrm{N}=1)$

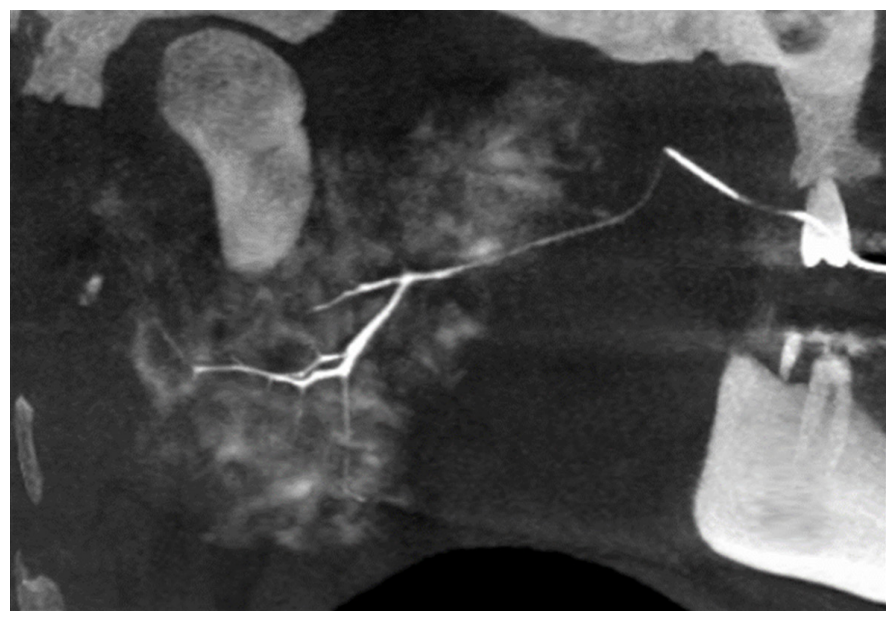

Figure 2. Retained ICM in the salivary glands following sialo-CBCT

Gland image showing parotid Sialo-CBCT of a female patient (41 yrs) with dry mouth sensation. Sialo-CBCT demonstrated ductopenia (showing only primary and secondary ducts) and marked extravasation of ICM into the gland parenchyma. and atrophy $(\mathrm{N}=1)$. Of note, most of the patients disclosed concomitant medical conditions that are associated with dry mouth symptoms rather than with risk factors for allergy. The list of co-moribidities included diseases such as fibromyalgia, rheumatoid arthritis, graft-versus host disease, and malignancies.

\section{Discussion}

To the best of our knowledge, this is the largest study thus far on IMC allergy in sialography. We did not detect a single allergic reaction in 1,515 patients, including 13 subjects with a previous diagnosis of ICM allergy. In comparison, our previous study on ICM allergy following coronary angiography found 3 reactions among 963 procedures. ${ }^{10}$ There are several potential explanations for the rarity of hypersensitivity reactions during sialography. The volume of injected ICM is approximately one tenth or less of that injected during procedures such as CT scan or coronary angiography. Furthermore, systemic exposure is low due to introduction of the media into a salivary duct, rather than intravenously. This is followed by clearance into the oral cavity and then to the gastrointestinal tract, where absorption is insignificant. Systemic absorption is ostensibly low even in the presence of ICM retention or extravasation. Therefore, we propose that ICM allergy is very unlikely when the agent is not injected systemically to a vein or an artery and pre-existing factors may not increase the risk for reactions considerably.

It is also noteworthy that patients are often regarded erroneously as ICM-allergic. In this study we found that in 7 of the 13 subjects a justification for this diagnosis could either not be found, or that it was based on misinterpretation. 
This is in line with our previous report that in approximately half of the patients, the reason for having a title of ICM allergy is obscure. ${ }^{10}$ It is well acknowledged that ICM allergy is often confused with sea-food or fish allergy, which represents an immune response to food protein. ${ }^{11}$ The source of this mistake is unclear but is probably related to the fact that both contrast media and these foods contain iodine. However, iodine per-se is an atom that does not induce allergic reactions and therefore the term "iodine allergy" is misleading and unjustifiable. False diagnosis of allergy is a widely recognized problem with regards to other drugs as well, most notably antibiotics. It is known to have a harmful impact on patient wellbeing and prognosis. In the case of ICM, it may cause delays or cancellations of well-indicated imaging or treatment procedures. Alternatively, patients may be exposed to excessive and unnecessary premedication, including corticosteroids. These arguments strongly suggest that over-estimation of allergy may potentially be at least as deleterious as unrecognized risk factors for future reactions.

The prevalence of bona fide allergic response to ICM has considerably decreased since the advent of new generation contrast agents. However, it appears that clinical practice has not sufficiently adapted to these improvements. Consequently, sialography is still not provided to patients suspected to have ICM allergy. ${ }^{12}$ The weakness of this study, however, is that the exact prevalence in ICM allergy during sialography could not be determined due to the lack of reaction events. Furthermore, the retrospective methodology does not allow confirmation the data or its completeness as reported in the medical records.

In summary, we present data from 1,515 subjects who underwent sialography, including 13 with a history of ICM allergy, most of whom did not receive premedication. No allergic reactions were found in the entire study population, demonstrating that ICM allergy is exceedingly uncommon in this setting. Therefore, we conclude that suspected allergy should not be regarded as a contra-indication for sialography. Furthermore, the findings reported herein strongly suggest that pre-medication is unnecessary in the vast majority of patients. The study did not include subjects with a clear history of severe allergic reactions to ICM and, therefore, our conclusions may not apply to such cases.

\section{References}

1. Hasson O. Modern sialography for screening of salivary gland obstruction. J Oral Maxillofac Surg. 2010;68(2):276-80.

2. Sobrino-Guijarro B, Cascarini L, Lingam RK. Advances in imaging of obstructed salivary glands can improve diagnostic outcomes. Oral Maxillofac Surg. 2013;17(1):11-9.

3. Keshet N, Aricha A, Friedlander-Barenboim S, Aframian DJ, Nadler C. Novel parotid sialo-cone-beam computerized tomography features in patients with suspected Sjogren's syndrome. Oral Dis. 2019;25(1):126-32.

4. Bertin H, Bonnet R, Delemazure AS, Mourrain-Langlois E, Mercier J, Corre P. Three-dimensional cone-beam CT sialography in non tumour salivary pathologies: procedure and results. Dentomaxillofac Radiol. 2017;46(1):20150431.

5. Abdel-Wahed N, Amer ME, Abo-Taleb NSM. Assessment of the role of cone beam computed sialography in diagnosing salivary gland lesions, Imaging Sci Dent. 2013;43(1):17-23.

6. American College of Radiology Committee on Drugs and Contrast Media. ACR Manual on Contrast Media. American College of Radiology, Version 2020. https://www.acr.org/Clinical-Resources/Contrast-Manual.

7. Salerno S, Cannizzaro F, Lo Casto A, Speciale R. Late allergic reaction following sialography. Dentomaxillofac Radiol. 2002;31(2):154.

8. Cockrell DJ, Rout PGJ. An adverse reaction following sialography. Dentomaxillofac Radiol. 1993;22(1):41-2.

9. Afzelius P, Nielsen MY, Ewertsen C, Bloch KP. Imaging of the major salivary glands. Clin Physiol Funct Imaging. 2016;36(1):1-10.

10. Topaz G, Karas A, Kassem N, Kitay-Cohen Y, Pereg D, Shilo L, et al Iodinated Contrast Media Allergy in Patients Hospitalized for Investigation of Chest Pain. J Allergy Clin Immunol Pract. 2018;6(6):2059-64.

11. Böhm I, Morelli J, Nairz K, Silva Hasembank Keller P, Heverhagen JT. Myths and misconceptions concerning contrast media-induced anaphylaxis: a narrative review. Postgrad Med. 2017;129(2):259-66.

12. Erdoğan NK, Altay C, Özenler N, Bozkurt T, Uluç E, Mete BD, et al. Magnetic resonance sialography findings of submandibular ducts imaging. Biomed Res Int. 2013;2013:417052. 